\title{
Évaluation de l'intérêt d'une consultation post-urgence d'urologie : étude rétrospective sur trois ans
}

\section{Usefulness of a Urology Clinic Post-Emergency Department: a 3-Year Retrospective Study}

\author{
M. Cortial · B.-I. Mewasing · M. Tligui · S. Dautheville $\cdot$ M. Valentian $\cdot$ A. Mintandjian $\cdot$ P. Ray \\ Reçu le 4 octobre 2017; accepté le 6 décembre 2017 \\ (C) SFMU et Lavoisier SAS 2017
}

Résumé Introduction : L'objectif principal de ce travail était d'évaluer l'intérêt de la consultation post-urgence d'urologie (CPUU) mise en place dans notre institution.

Patients et méthodes : Il s'agit d'une étude transversale, monocentrique et rétrospective sur trois années distinctes : 2014, 2015 et 2016 de trois mois consécutifs (janvier à mars). Seuls les patients de plus de 18 ans ayant consulté aux urgences pour un motif urologique et relevant de la CPUU ont été inclus.

Résultats : Au total, 465 patients ont été programmés en CPUU, dont $265(57 \%)$ se sont présentés à la consultation. Deux cent vingt-deux patients $(48 \%)$ ont finalement répondu aux critères d'inclusion. Les principaux diagnostics relevant de la CPUU étaient principalement les coliques néphrétiques ( $45 \%)$, la rétention aiguë d'urine $(14 \%)$ et les infections de l'appareil urinaire (16\%). On note une diminution significative du délai de CPUU passant à moins de deux semaines pour $60 \%$ des patients en 2016 contre 30 et $38 \%$ respectivement en 2014 et $2015(p=0,003)$. Le nombre de modifications diagnostiques à la sortie de la CPUU reste faible (6\% sur les trois ans ; $p=0,94)$. La modification thérapeutique par les urologues était principalement chirurgicale $(22 \% ; p=0,75)$. La CPUU a permis d'instaurer un suivi au long cours pour plus de la moitié des patients $(60 \% ; p=0,31)$.

\footnotetext{
M. Cortial · B.-I. Mewasing $\cdot$ S. Dautheville $\cdot$ M. Valentian

A. Mintandjian · P. Ray $(\bowtie)$

Service d'accueil des urgences, hôpital Tenon,

55, rue Pelleport, F-75020 Paris, France

e-mail : patrick.ray@tnn.aphp.fr

M. Tligui

Service d'urologie, hôpital Tenon,

55, rue Pelleport, F-75020 Paris, France

P. Ray

Sorbonne université, DHU FAST (Fight Against Stress),

75013 Paris
}

Conclusion : La CPUU semble démontrer un intérêt tant sur le plan diagnostique que sur le plan du suivi spécialisé dans notre centre.

Mots clés Urgences urologiques · Colique néphrétique · Consultation post-urgence

\begin{abstract}
Introduction: The main objective of this work was to evaluate the usefulness of a post-emergency urology clinic (PEUC).

Patients and methods: This was a monocentric, observational, descriptive and retrospective study over three consecutive months (January to March) of three distinct years: 2014, 2015 and 2016. Only patients over 18 years of age who consulted the emergency department for a urological disease were included in the PEUC.

Results: A total number of 465 patients were scheduled for a PEUC and out of which only 265 patients (57\%) attended the consultation. 222 patients ( $48 \%$ ) finally met the inclusion criteria. The most frequent urological emergencies seen in PEUC were renal colic (RC) (45\%), acute urine retention $(14 \%)$ and urinary tract infections $(16 \%)$ without any difference over the years. There was a significant decrease in the PEUC timeframe over the years: less than 2 weeks for $60 \%$ of patients in 2016 compared with $30 \%$ and $38 \%$ in 2014 and 2015 , respectively $(P=0.003)$. The percentage of diagnosis modification by the urologists remained low ( $6 \%$ over three years, $P=0.94)$. Treatment modification was mainly surgical $(22 \% ; P=0.75)$. The PEUC has enabled follow-up for more than $50 \%$ of patients who had been enrolled over time $(60 \% ; P=0.31)$.

Conclusion: The PEUC seems to demonstrate usefulness both in terms of diagnosis and appropriate treatment with mid-term follow-up.
\end{abstract}

Keywords Urological emergencies · Renal colic · Post-emergency consultation 


\section{Introduction}

En France, les consultations d'urologie aux urgences sont, en moyenne, de cinq par jour pour 100 patients consultant, et les hospitalisations en urgence représentent $8 \%$ de l'ensemble des hospitalisations d'urologie [1-2].

L'intérêt des consultations post-urgence (CPU) est suggéré au regard du long délai de rendez-vous qui existe pour certaines spécialités et qui n'est pas adapté à la demande des patients consultant aux urgences adultes. Ce délai reste, selon l'enquête IFOP réalisée en 2016, la principale difficulté d'accès aux soins [3]. Les études concernant les CPU sont peu nombreuses. Bien que ces études soient pratiquement toutes observationnelles et dotées de faibles cohortes, elles ont tout de même mis en évidence un avantage médicoéconomique en proposant un avis spécialisé rapide aux patients [4-7], mais permettent également une réévaluation diagnostique, clinique et thérapeutique rapide par le spécialiste. En plus de répondre à un vrai besoin et à une demande de suivi, la CPU s'inscrit dans l'optique d'une meilleure organisation des urgences au vu du nombre croissant de passages aux urgences et de la diminution des lits d'aval. En effet, un certain nombre de patients sont adressés aux urgences pour faciliter l'accès aux avis spécialisés. Nous avons déjà suggéré l'intérêt de ce type de consultation en pneumologie [4]. L'hôpital Tenon fait partie des huit services d'urologie pour adultes appartenant à l'AP-HP en Île-de-France pour répondre à un besoin de proximité grandissant. La CPU d'urologie (CPUU) a été initialement créée en 2013 pour le suivi des patients présentant une colique néphrétique $(\mathrm{CN})$, pris en charge en ambulatoire, puis a rapidement été élargie aux patients relevant d'une urgence urologique.

L'objectif principal de notre travail est d'évaluer l'intérêt d'une CPUU ainsi que la typologie de la patientèle concernée sur une période de trois ans. L'objectif secondaire est d'évaluer si la gestion des $\mathrm{CN}$ aux urgences est conforme aux recommandations réactualisées de 2008 [8].

\section{Patients et méthode}

\section{Design de l'étude}

Ce travail est une étude transversale, monocentrique et rétrospective. Il a été conduit dans un centre hospitalouniversitaire parisien : l'hôpital Tenon (44 000 passages d'adultes par an). Dans notre hôpital, un interne d'urologie est de garde sur place, avec un senior d'astreinte. Sur le plan éthique, cette étude était hors du champ de la loi Jardé de par son caractère rétrospectif.

Une feuille standardisée de demande de CPUU a été élaborée par les urgentistes et les urologues. Sur cette feuille figurent : le nom, le prénom, le numéro de téléphone du patient ; ceux de son médecin traitant, le nom de l'urgentiste qui a pris en charge le patient, le diagnostic supposé et le délai de consultation désiré. Ce bon de demande de CPUU est rempli par le médecin urgentiste, puis faxé au secrétariat de la CPUU. Les patients sont contactés par l'urologue référent de la CPUU dans les 48-72 heures. Ce dernier prévoit le rendez-vous de consultation en fonction du diagnostic et des examens complémentaires. Deux demi-journées par semaine sont dédiées à la CPUU, avec en moyenne une dizaine de patients par semaine.

Tout patient de plus de 18 ans, ayant consulté au service d'accueil des urgences (SAU) de Tenon pour un motif urologique pour lequel la prise en charge a été ambulatoire et ayant bénéficié de la CPUU, a été inclus. Les patients inclus étaient identifiés à partir d'une liste de patients provenant des urgences, pour lesquels une demande de CPUU a été faxée à la secrétaire responsable de cette consultation. Ont été exclus les patients mineurs ou n'ayant pas préalablement consulté aux urgences, ou ne s'étant pas présentés à la CPUU, et/ou ceux dont les dossiers étaient incomplets ou non retrouvés. Les données ont été extraites sur trois mois consécutifs de janvier à mars, mais sur trois années distinctes : 2014, 2015 et 2016. Le choix des trois mois s'est fait de manière arbitraire, et celui des trois ans a eu pour but l'évaluation et la typologie de l'activité de la CPUU et, par ailleurs, l'évolution des pratiques des urgentistes dans la prise en charge des urgences urologiques, en particulier pour les $\mathrm{CN}$, tant sur le plan diagnostique que thérapeutique. Cette évolution au cours du temps a permis également de vérifier l'adéquation aux recommandations, pour la prise en charge des $\mathrm{CN}$.

La récupération de données des patients incluables s'est faite par le biais de deux logiciels informatiques : celui du SAU, UrQual ${ }^{\circledR}$ (Maincare Solutions, Cestat, France) et Mediweb $^{\circledR}$ (AP-HP, France) qui est le principal portail d'accès à l'information médicale. Les données recueillies par le biais d'UrQual ${ }^{\circledR}$ comprenaient les variables d'ordre épidémiologique (l'âge, le sexe, les antécédents), clinique (le motif de consultation et les pathologies diagnostiquées) et thérapeutique (la prise en charge et le devenir du patient). Puis, le délai de consultation, le diagnostic définitif, la modification de prise en charge et le suivi des patients après leur CPUU ont ainsi pu être récupérés et analysés à partir du logiciel Mediweb ${ }^{\circledR}$.

\section{Analyse statistique}

Les comparaisons statistiques ont été effectuées en situation bilatérale, puis en pourcentage, pour ensuite être comparées en utilisant le test « exact de Fisher ». Les données continues ont été résumées par leur moyenne et l'écart-type, par le biais du logiciel Excel. L'égalité de population ainsi que les variables quantitatives ont été traitées en utilisant le test non paramétrique de Kruskal-Wallis, du fait de la comparaison de 
plus de deux populations et de l'effectif faible de patients. Une valeur de $p$ inférieure à 0,05 a été considérée comme statistiquement significative.

\section{Résultats}

Quatre cent soixante-cinq demandes ont été faxées durant le premier trimestre des trois années respectives, dont 131 en 2014, 175 en 2015 et 159 en 2016 (Fig. 1). Cela représente $2 \%$ des patients consultant au SAU de Tenon. $\mathrm{Au}$ total, 265 sur 465 patients (57\%) convoqués se sont présentés à la CPUU, dont 222 (48 \%) inclus au final dans notre étude. Cinquante-neuf pour cent des patients ont été revus en moins de deux semaines en 2016, contre $42 \%$ en 2015 et $30 \%$ en $2014(p=0,003)$.

Les caractéristiques démographiques étaient identiques entre les trois phases sur les trois années étudiées (Tableau 1). La CN est l'antécédent urologique le plus fréquemment retrouvé. Le diagnostic de CN était la pathologie la plus fréquemment retrouvée, quelle que soit l'année étudiée. Le diagnostic de rétention aiguë d'urine (RAU) a augmenté au fil des années. Les autres diagnostics initiaux supposés comprenaient l'hématurie et les problèmes de matériel (sonde

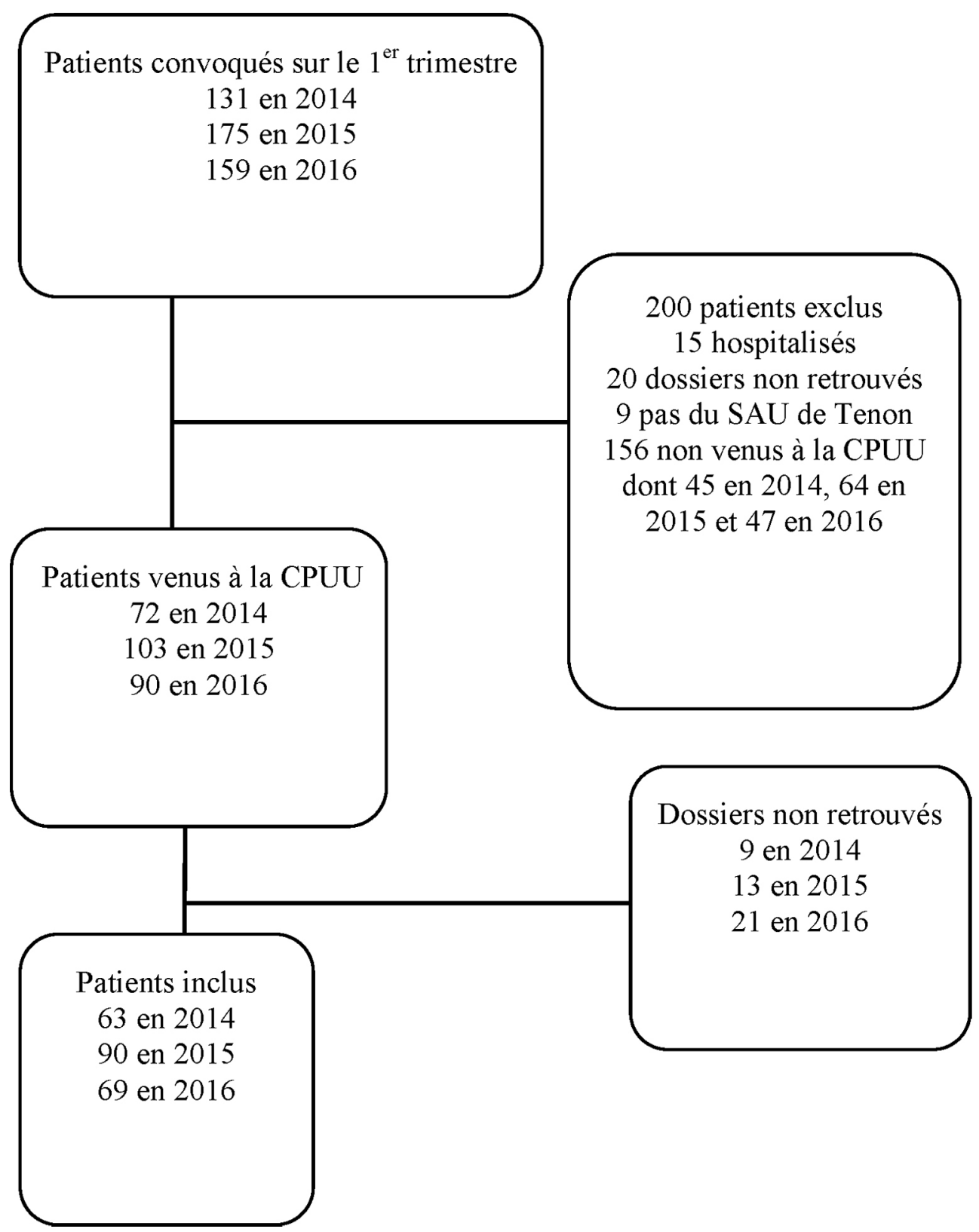

Fig. 1 Diagramme de flux. CPUU : consultation post-urgence d'urologie ; SAU : service d'accueil des urgences 
urinaire, cathéter sus-pubien, néphrostomie). Moins de $10 \%$ des diagnostics initiaux ont été modifiés par les urologues sur les trois années $(p=0,94)$ (Tableau 2). Quarante-huit patients ont eu une modification de leur traitement initial (Tableau 3). En excluant les CN, la prise en charge de ces patients a été exclusivement chirurgicale. Les urologues ont modifié le diagnostic pour seulement six patients dans notre étude (Tableau 2). Les urologues ont modifié le traitement de la $\mathrm{CN}$ pour 22 patients. En effet, il s'agissait soit d'une $\mathrm{CN}$ résistante au traitement du fait de la taille du calcul, soit compliquée d'une dégradation de la fonction rénale : 15 patients ont eu une dérivation des urines par la pose de sonde double $J$, et 7 ont eu une séance de lithotritie extracorporelle. Les patients ont été par la suite suivis par les urologues dans le cadre du bilan métabolique.
Notre objectif secondaire était d'évaluer la prise en charge diagnostique et thérapeutique de la $\mathrm{CN}$ prise en charge en ambulatoire par les urgentistes, par rapport aux recommandations publiées en 2008. Globalement, la prise en charge de la $\mathrm{CN}$ dans notre service semble adaptée (Tableau 4), avec un dosage de la créatininémie et la prescription d'AINS très fréquemment.

\section{Discussion}

Nous avons voulu évaluer l'intérêt de la CPUU et ainsi dresser un tableau de la typologie des patients ayant relevé de cette CPUU mise en place il y a quelques années dans notre structure. Plus de $50 \%$ des patients relevant de la CPUU ont

Tableau 1 Caractéristiques des patients

\begin{tabular}{|c|c|c|c|c|}
\hline & 2014 & 2015 & 2016 & Valeur de $p$ \\
\hline Nombre de patients & 63 & 90 & 69 & \\
\hline Âge & $48 \pm 20$ & $49 \pm 20$ & $44 \pm 19$ & 0,36 \\
\hline Sexe masculin & $47(75)$ & $73(81)$ & $56(81)$ & 0,56 \\
\hline \multicolumn{5}{|l|}{ Antécédents } \\
\hline Aucun & $32(51)$ & $44(49)$ & $30(44)$ & 0,68 \\
\hline Colique néphrétique & $15(24)$ & $27(30)$ & $13(19)$ & 0,27 \\
\hline Infections & $2(3)$ & $3(3)$ & $1(2)$ & 0,77 \\
\hline Adénome prostatique & $5(8)$ & $8(9)$ & $11(16)$ & 0,25 \\
\hline Autres & $14(14)$ & $16(9)$ & $25(20)$ & 0,03 \\
\hline \multicolumn{5}{|l|}{ Diagnostic initial } \\
\hline Colique néphrétique & $32(51)$ & $41(46)$ & $27(39)$ & 0,40 \\
\hline Infections & $9(14)$ & $20(22)$ & $7(10)$ & 0,12 \\
\hline RAU & $3(5)$ & $11(12)$ & $17(25)$ & 0,004 \\
\hline Douleur organique & $9(14)$ & $8(9)$ & $7(10)$ & 0,56 \\
\hline Autres & $10(16)$ & $10(11)$ & $11(16)$ & 0,57 \\
\hline Diagnostic modifié & $4(6)$ & $5(6)$ & $5(7)$ & 0,94 \\
\hline Traitement modifié & $12(19)$ & $20(22)$ & $17(25)$ & 0,75 \\
\hline Suivis au long cours & $42(67)$ & $53(59)$ & $37(54)$ & 0,31 \\
\hline
\end{tabular}

Tableau 2 Modification des diagnostics

\begin{tabular}{|lll|}
\hline Années & Diagnostic initial erroné & Diagnostic modifié final \\
\hline 2014 & 4 coliques néphrétiques & 1 pathologie digestive \\
2015 & 1 colique néphrétique & 3 infections urinaires \\
& 1 rétention aiguë d'urine & 1 pathologie digestive \\
& 1 hématurie & 1 colique néphrétique compliquée \\
& 2 infections & 1 cancer \\
& 1 colique néphrétique & 1 cancer et 1 douleur organique \\
& 2 rétentions aiguës d'urine & 1 lumbago \\
& 2 hématuries & 2 coliques néphrétiques compliquées \\
& & 1 colique néphrétique compliquée et 1 infection \\
\hline
\end{tabular}


bénéficié d'un suivi urologique au long cours répondant probablement à un réel besoin de prise en charge spécialisée de notre bassin de population. Les deux principaux diagnostics sont la $\mathrm{CN}$ et la RAU, ce qui est concordant avec la littéra-

\begin{tabular}{|ll|}
\hline $\begin{array}{l}\text { Tableau } 3 \\
\text { modifié }\end{array}$ & Pathologies pour lesquelles le traitement initial a été \\
\hline Années & Pathologies \\
\hline $2014 \quad$ & 5 coliques néphrétiques \\
& 4 douleurs organiques (varicocèles) \\
& 3 hématuries \\
& 10 coliques néphrétiques \\
& 1 anomalie anatomique \\
& 1 rétention aiguë d'urine \\
& 5 douleurs organiques (varicocèles) \\
& 3 hématuries \\
& 7 coliques néphrétiques \\
& 2 anomalies anatomiques \\
& 4 rétentions aiguës d'urines \\
& 2 douleurs organiques (1 varicocèle et 1 rupture \\
du rein) & \\
& 1 hématurie
\end{tabular}

ture $[1,2,9,10]$. On note une augmentation significative de la RAU, au fil des années, relevant de la CPUU. Cela s'explique possiblement par le fait que la RAU se gérait auparavant en hospitalier, alors qu'au fur et à mesure, la prise en charge est devenue ambulatoire. Le pourcentage de modification diagnostique à la sortie de la CPUU par les urologues est faible. Cela nous conforte dans notre prise en charge des urgences urologiques au SAU. Ainsi, les modifications de traitement ont surtout été chirurgicales $(\mathrm{CN})$ et ont été posées sur les facteurs évolutifs cliniques ou révélateurs de la pathologie par de l'imagerie. Plus de la moitié des patients vus en CPUU ont bénéficié d'un suivi au long cours, ce qui est un argument pour l'utilité de cette CPUU. Cela permet un parcours de soins ambulatoire et une prise en charge spécialisée. La CPUU pourrait avoir diminué le taux de reconsultation, mais évidemment cela n'est pas démontré par notre travail. Seulement $60 \%$ des patients se sont présentés en CPUU. Néanmoins, ce taux est très supérieur à celui retrouvé dans d'autres études sur les CPU (23\%) et notamment dans la CPU de pneumologie de notre hôpital [4]. Le fax de la demande de CPUU est souvent donné au patient à sa sortie, et le fait qu'un chirurgien urologue rappelle lui-même les patients pourrait jouer un rôle dans ce taux élevé de présence à la CPUU. Il serait important de travailler à améliorer

\begin{tabular}{|c|c|c|c|c|}
\hline & $2014(n=15)$ & $2015(n=27)$ & $2016(n=13)$ & Valeur de $p$ \\
\hline Âge & $35 \pm 13$ & $44 \pm 12$ & $40 \pm 15$ & 0,21 \\
\hline Sexe masculin & $18(56)$ & $30(73)$ & $16(59)$ & 0,27 \\
\hline Antécédents de CN & $15(47)$ & $15(37)$ & $11(41)$ & 0,67 \\
\hline Avis spécialisé au SAU & $5(16)$ & $6(15)$ & $6(22)$ & 0,75 \\
\hline \multicolumn{5}{|l|}{ Examens au SAU } \\
\hline $\mathrm{BU}$ & $32(100)$ & $41(100)$ & $27(100)$ & 1 \\
\hline Hématurie & $24(75)$ & $34(83)$ & $23(85)$ & 0,65 \\
\hline Biologie & $29(91)$ & $31(76)$ & $25(93)$ & 0,09 \\
\hline Échographie & $2(6)$ & $4(10)$ & $4(15)$ & 0,58 \\
\hline Scanner & $7(22)$ & $4(10)$ & $8(30)$ & 0,09 \\
\hline \multicolumn{5}{|l|}{ Examens en ville } \\
\hline Biologie & $3(9)$ & $7(17)$ & $6(22)$ & 0,42 \\
\hline Échographie & $6(19)$ & $11(26)$ & $4(15)$ & 0,50 \\
\hline Scanner & $18(56)$ & $20(49)$ & $13(48)$ & 0,77 \\
\hline $\begin{array}{l}\text { Traitement de sortie } \\
\text { AINS }\end{array}$ & $29(94)$ & $34(87,2)$ & $24(89)$ & 0,68 \\
\hline Alphabloquant & $4(13)$ & $10(24)$ & $7(26)$ & 0,37 \\
\hline Diagnostic modifié & $4(13)$ & $1(2)$ & $1(4)$ & 0,23 \\
\hline Traitement modifié & $5(16)$ & $10(24)$ & $7(26)$ & 0,60 \\
\hline Suivi & $24(75)$ & $25(61)$ & $12(44)$ & 0,06 \\
\hline
\end{tabular}


encore ce taux par le rappel de la consultation en envoyant un e-mail ou un SMS ou par un rappel téléphonique la veille du rendez-vous.

L'objectif secondaire de notre étude a été d'évaluer les pratiques des urgentistes dans la prise en charge des $\mathrm{CN}$ et de vérifier leur conformité aux recommandations [8]. Notre patientèle est une population jeune et masculine, avec des antécédents de $\mathrm{CN}$ dans presque la moitié des cas, ce que confirment les études [11-14]. La pratique d'une bandelette urinaire est un examen facile et peu coûteux, et dans notre travail, la bandelette urinaire a été réalisée chez $100 \%$ des patients. Mais l'hématurie n'a été retrouvée que chez $80 \%$ des patients, en concordance avec la littérature [15-17]. Contrairement aux recommandations de la SFMU et à celles de l'European Association of Urology (grade C) [18], dans notre étude, la créatininémie n'est pas systématiquement prescrite $(87 \%)$. La prise en charge des $\mathrm{CN}$ impose la réalisation d'une imagerie soit au SAU pour celles qui sont compliquées, ou en ambulatoire pour celles qui sont a priori non compliquées. Le choix de l'imagerie entre l'échographie et la tomodensitométrie (TDM) non injectée low dose dépend directement du médecin prescripteur. Les recommandations françaises penchent pour la réalisation d'une échographie couplée à un ASP en première intention (grade A) [8], favorisée aussi par son caractère peu irradiant. Dans notre institution, la TDM low dose est l'examen radiologique le plus prescrit en première intention, car cela est une préférence de nos urologues. Il semble que la TDM low dose soit une tendance nationale, voire internationale [19-21]. On note par ailleurs une augmentation - certes non significative des prescriptions d'imagerie au SAU en 2016 par rapport à 2014 et à 2015. Néanmoins, la réalisation de l'échographie au lit du patient par l'urgentiste pourrait faciliter la prise en charge des $\mathrm{CN}$ [19]. Le diagnostic a été erroné chez six patients dans notre étude (Tableau 2), sans différence significative entre les trois années $(p=0,23)$, et l'imagerie a permis de trancher en faveur de pathologies digestive, infectieuse et rachidienne. Dans notre travail, les urologues ont modifié le traitement de la $\mathrm{CN}$ instauré au SAU pour 22 patients dans notre étude. Cependant, le nouveau traitement instauré était soit chirurgical, par dérivation des urines, soit la lithotritie extracorporelle. Il semble donc que la gestion des CN au SAU Tenon soit en accord avec les recommandations.

L'étude était monocentrique, rétrospective et non exhaustive, avec des biais inhérents à ce type de méthodologie. Les patients inclus dans cette étude ne peuvent pas être représentatifs de la population générale, et nos conclusions ne peuvent être extrapolées à d'autres structures, d'autant que seuls $60 \%$ des patients se sont présentés en consultation et qu'il s'agit d'un hôpital avec une spécificité en uronéphrologie. Il faut souligner que les modifications thérapeutiques réalisées par l'urologue ne remettent pas forcément le diagnostic de l'urgentiste en cause. Par exemple, le patient peut consulter pour hématurie et être adressé à la CPUU pour la suite, c'està-dire que la cystoscopie doit être organisée et réalisée par le chirurgien, pour un diagnostic final du cancer urothélial diagnostiqué après la CPUU. Dans ce cas, la modification peut avoir été prévue et anticipée par l'urgentiste qui, en l'adressant en CPPU, a planifié sa réalisation. Malheureusement, nous ne pouvons pas vérifier toutes ces situations déjà prévues dans l'esprit de l'urgentiste, car les dossiers de sortie ne comportaient pas de précisions. Nous n'avons pas évalué les différents antalgiques administrés en fonction de l'EVA ainsi que la posologie des analgésiques pour la prise en charge médicamenteuse de la CN. Il aurait été intéressant de voir le nombre de patients qui ont consulté au SAU pendant la période d'inclusion et qui auraient pu relever de la CPUU. Enfin, nous n'avons pas évalué la satisfaction des patients ou des urgentistes ni celle des urologues.

\section{Conclusion}

Notre étude suggère l'intérêt de la CPUU dans la prise en charge diagnostique mais également thérapeutique des patients. Elle a permis la rectification rapide de certains diagnostics et de leur prise en charge thérapeutique et la mise en place d'un suivi pour des patients relevant d'une pathologie chronique urologique.

Liens d'intérêts : les auteurs déclarent ne pas avoir de lien d'intérêt.

\section{Références}

1. Martin L, Pillot P, Bardonnaud N, et al (2013) Évaluation de l'activité d'une unité d'urgences urologiques en centre hospitalier universitaire. Prog Urol 24:62-6

2. Mondet F, Chartier-Kastler E, Yonneau L, et al (2002) Épidémiologie des urgences urologiques en centre hospitalier universitaire. Prog Urol 12:437-42

3. IFOP-JALMA. L'Observatoire de l'accès aux soins, enquête menée auprès des Français et des professionnels de santé. http:// www.leciss.org/sites/default/files/111030_Enquete_IFOP-JALMA_ nov2011.pdf (Dernier accès le 22 mai 2017)

4. Valentian M, Naccache JM, Cadranel J, et al (2016) Intérêt de la mise en place d'une consultation post-urgence de pneumologie : évaluation à 21 mois. Ann Fr Med Urgence 6:93-8

5. Barge S, Boubaker B, Chitic G, et al (2015) La consultation posturgence en hépatogastroentérologie contribue-t-elle à améliorer l'aval des urgences ? Résumé présenté aux Journées francophones d'hépatogastroentérologie et d'oncologie digestive. Paris, France. http://www.snfge.org/content/la-consultation-post-urgenceen-hepato-gastroen (Dernier accès le 25 août 2017)

6. Ingen-Housz-Oro S, Mirkamali A, Valeyrie-Allanore L, et al (2010) Activity report on a rapid post-emergency surgery for the management of dermatological emergencies. Ann Dermatol Venereol 137:519-22 
7. Lekouky (2009) Consultation traumatologique post-urgences au CHU Saint-Antoine : étude descriptive et comparative en fonction du parcours de soins : place accordée par les consultants à la médecine ambulatoire en traumatologie (thèse d'exercice, faculté de médecine de Paris-VII-Diderot) (Dernier accès le 27 juin 2017)

8. El Khebir M, Fougeras O, LeGall C, et al (2009) Actualisation 2008 de la $8^{\mathrm{e}}$ Conférence de consensus de la Société francophone d'urgences médicales de 1999. Prise en charge des coliques néphrétiques de l'adulte dans les services d'accueil et d'urgences. Prog Urol 19:462-73

9. Diallo MB, Bah I, Baldé I (1996) Les urgences urologiques au CHU Ignace-Deen, étude rétrospective. Guin Med 10:9-14

10. Fall B, Diao B, Fall PA, et al (2008) Les urgences urologiques en milieu hospitalier universitaire à Dakar : aspects épidémiologiques, cliniques et thérapeutiques. Prog Urol 18:650-3

11. Afshar K, Jafari S, Marks AJ, et al (2015) Nonsteroidal antiinflammatory drugs (NSAIDs) and non-opioids for acute renal colic. Cochrane Database Syst Rev 6:CD006027

12. Pathan SA, Mitra B, Straney LD, et al (2016) Delivering safe and effective analgesia for management of renal colic in the emergency department: a double-blind, multigroup, randomised controlled trial. Lancet 387:1999-2007

13. Wang RC, Smith-Bindman R, Whitaker E, et al (2017) Effect of tamsulosin on stone passage for ureteral stones: a systematic review and meta-analysis. Ann Emerg Med 69:353-61
14. Bounes V, Vallé B, Concina F, et al (2016) Treatment of acute renal colic in US and French EDs: simulated cases and real cases in acute pain management. Am J Emerg Med 34:1955

15. Xafis K, Thalmann G, Benneker LM, et al (2008) Forget the blood, not the stone! Microhaematuria in acute urolithiasis and the role of early CT scanning. Emerg Med J 25:640-4

16. Kobayashi T, Nishizawa K, Mitsumori K, Ogura K (2003) Impact of date of onset on the absence of hematuria in patients with acute renal colic. J Urol 170:1093-6

17. Bove P, Kaplan D, Dalrymple N, et al (1999) Reexamining the value of hematuria testing in patients with acute flank pain. J Urol 162:685-7

18. Tiselius HG, Ackermann DK, Alken P, et al (2008) Guidelines on urolithiasis. European Association of Urology. http://uroweb.org/ wp-content/uploads/22-Urolithiasis_LR_full.pdf (Dernier accès le 13 mai 2017)

19. Smith-Bindman R, Aubin C, Bailitz J, et al (2014) Ultrasonography versus computed tomography for suspected nephrolithiasis. N Engl J Med 371:1100-10

20. Broder J, Bowen J, Lohr J, et al (2007) Cumulative CT exposures in emergency department patients evaluated for suspected renal colic. J Emerg Med 33:161-8

21. Schoenfeld EM, Pekow PS, Shieh MS, et al (2017) The diagnosis and management of patients with renal colic across a sample of US hospitals: high CT utilization despite low rates of admission and inpatient urologic intervention. PLoS One 12:e0169160 